\title{
Evaluation of the mobile phone electromagnetic radiation on serum iron parameters in rats
}

\author{
Murat Çetkin ${ }^{1}$, Can Demirel ${ }^{2}$, Neşe Kızılkan ${ }^{3}$, Nur Aksoy ${ }^{4}$, Hülya Erbağc1 ${ }^{4}$
}

\author{
1. Department of Physical Therapy and Rehabilitation, Faculty of Health Science, European \\ University of Lefke, Lefke, TRNC \\ 2. Department of Biophysics, Faculty of Medicine, Gaziantep University, Gaziantep, Turkey \\ 3. Department of Anatomy, Faculty of Medicine, Gaziantep University, Gaziantep, Turkey \\ 4. Gaziantep/Şehitkamil
}

\begin{abstract}
Background: Electromagnetic fields (EMF) created by mobile phones during communication have harmful effects on different organs.

Objectives: It was aimed to investigate the effects of an EMF created by a mobile phone on serum iron level, ferritin, unsaturated iron binding capacity and total iron binding capacity within a rat experiment model.

Methods: A total of 32 male Wistar albino rats were randomly divided into the control, sham, mobile phone speech (2h/day) and stand by $(12 \mathrm{~h} /$ day $)$ groups. The speech and stand by groups were subjected to the EMF for a total of 10 weeks.

Results: No statistically significant difference was observed between the serum iron and ferritin values of the rats in the speech and stand by groups than the control and sham groups ( $p>0.05)$. The unsaturated iron binding capacity and total iron capacity values of the rats in the speech and stand by groups were significantly lower in comparison to the control group ( $p<0.01$ ).

Conclusion: It was found that exposure to EMF created by mobile phones affected unsaturated iron binding capacity and total iron binding capacity negatively.
\end{abstract}

Keywords: cellular phone, electromagnetic fields, ferritin, serum iron

DOI: https://dx.doi.org/10.4314/ahs.v17i1.23

Cite as: Çetkin M, Demirel C, Kıə̨lkean N, Aksoy N, Erbağcı H. Evaluation of the mobile phone electromagnetic radiation on serum iron parameters in rats. Afri Health Sci. 2017;17(1): 186-190. bttps:/ / dx.doi.org/10.4314/abs.v17i1.23

*This study was done in Biophysics and Anatomy Department of Medicine, Faculty of Gaziantep University

\section{Introduction}

Mobile phones have become our time's most popular communication devices with the help of the addition of new functions. Mobile phones work based on the transmission of radio frequency signals and they create an electromagnetic field (EMF) around them while doing $\mathrm{so}^{1}$. These devices have become widespread increased the concerns about the potentially harmful biological effects of the EMF they create. Previously, negative effects of EMF created by mobile phones were reported on hormones $^{2}$ and parameters such as hemoglobin, hematocrit

\section{Corresponding author: \\ Murat Çetkin, \\ Department of Physical Therapy and \\ Rehabilitation, Faculty of Health Science, \\ European University of Lefke, Lefke, TRNC \\ Email:muratcetkin@hotmail.com}

and red blood cells ${ }^{3}$. Additionally it was reported that exposure to EMF reduced the levels of blood serum elements such as magnesium, iron and copper ${ }^{4}$.

Iron is the one of serum element which has an important role in the active sites of a wide range of proteins involved in energy metabolism, respiration, and DNA synthesis ${ }^{5}$. While $65 \%$ of the iron in humans is bound to hemoglobin responsible for oxygen-carrying, $10 \%$ of it is found in the structures of myoglobin, cytochrome and enzymes that contain ions. $20-30 \%$ of the iron in the body is non-toxically stored as bound to ferritin and hemosiderin molecules. The transportation of the iron is achieved by the transferrin protein ${ }^{6}$. While transferring is a protein with high affinity and low capacity (each transferrin molecule is bound to two iron atoms), there are receptors on its surface for each cell that requires iron ${ }^{7}$. Unsaturated iron binding capacity (UIBC) is the measurement of the fraction of the serum transferrin which is not bound with 
iron $^{8}$ and it indicates the amount of iron that can be saturated with the free transferrin in the blood ${ }^{9}$. Total iron binding capacity (TIBC) indicates the maximum amount of iron essential to saturate serum transferrin ${ }^{10}$.

There are few studies in the literature on the effects of particularly the EMF created by mobile phones on serum iron parameters. Previously it was reported the EMF created by mobile phones affect the serum ferritin levels negatively ${ }^{11}$. In another study, it was observed that the serum iron and ferritin levels of people living close to high voltage electric cables which create EMF around were relatively lower ${ }^{12}$. Further experimental studies are needed in this field in order to clearly reveal the relationship between EMF and serum iron parameters.

In this study, it was aimed to investigate the effects of exposure to the EMF created by mobile phones on serum iron level, ferritin, UIBC and TIBC.

\section{Methods}

The experimental protocol was approved by Animal Experiments Local Ethics Committee decision numbered 11.2011/54-4 and dated 28.11.2011. All animals were kept in wire mesh cages for 12 hours at night and 12 hours in daytime, at circadian rhythms, $24-26^{\circ} \mathrm{C}$ ambient temperature and $45-60 \%$ humidity. A total of thirty-two $(n=32)$ male Wistar Albino rats were used in the study. These rats were 8-10 weeks old with $200 \pm 20 \mathrm{~g}$ weight. The rats were divided into four groups as the Control $(\mathrm{C} ; \mathrm{n}=8)$, Sham (Sh; $\mathrm{n}=8)$, mobile phone Speech (Sp; $\mathrm{n}=8)$ and mobile phone Stand by (S-by; $\mathrm{n}=8)$. The EMFs were generated by the mobile phones using GSM mobile phone systems. The properties of mobile phones were; the highest SAR value was $0,96 \mathrm{~W} / \mathrm{kg}$ and each of them had an 890-915 MHz carrier frequency band, $217 \mathrm{~Hz}$ modulation frequency, $250 \mathrm{~mW}$ maximum average power and $2 \mathrm{~W}$ maximum peak power ${ }^{13}$.
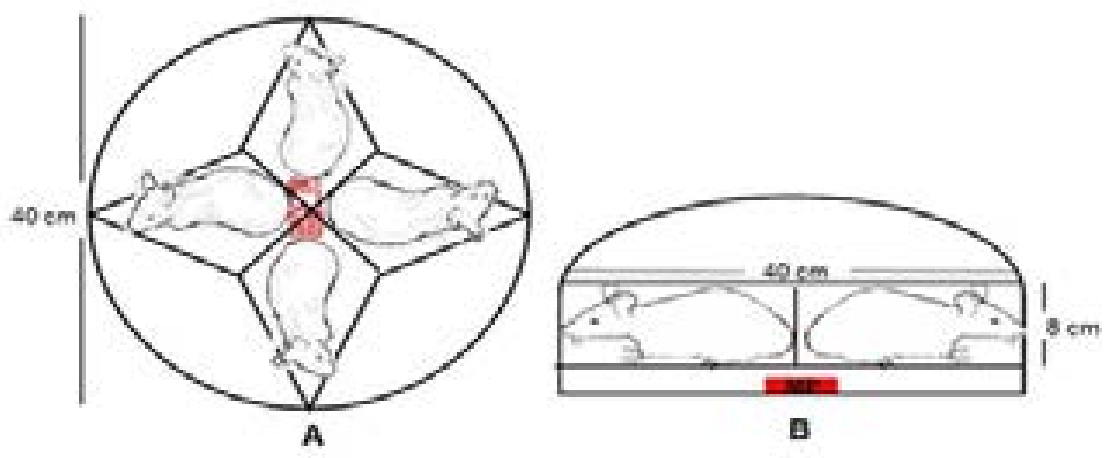

Schematic representation of the experimental set up on the A-top view and B- side view

Circular plastic experimental boxes were used in the Sh, Sp and S-by groups. One mobile phone was placed in the special sections located $1 \mathrm{~cm}$ below and in the middle of the experimental boxes (Figure 1).

The rats in group Sh were placed in the box with their testes directed towards the mobile phone while the mobile phones were switched off. The rats were kept in this experimental box for 12 hours/day. The rats in the Sp and S-by groups were placed in experimental boxes in the same way as those in group Sh. Speech mode was obtained by the mobile phone in speech mode for 2 hours/ day. The duration of standby mode was 12 hours/day. Experimental practices were carried out between 07:0019:00 for groups Sh and S-by and 07:00-09:00 for the Sp group seven days a week. The rats in the $\mathrm{C}$ group were kept in their normal life cages in a separate room without being exposed to any type of electromagnetic field. The duration of the experiment was 10 weeks for all groups.

\section{Evaluation of the serum iron parameters}

At the end of the experiment (on the last day of 10th week) all the rats were anesthetized by Ketamine-Hydrochloride $(50 \mathrm{mg} / \mathrm{kg}$ ) and Xylazine-Hydrochloride $(5 \mathrm{mg} /$ $\mathrm{kg}$ ) intraperitoneally. The anesthetization of rats in groups was made randomly. By thoracotomy, the thoracic cages of the rats were opened and blood samples were taken intracardially. Blood samples were drawn into common biochemical tubes and samples were centrifuged at 400RPM for 10 minutes and blood serums were obtained. Serum samples were stored at $-80{ }^{\circ} \mathrm{C}$ before the evaluations of serum iron parameters. The evaluated serum iron parameters were serum iron level, ferritin and UIBC. The total 
iron binding capacity was calculated by the sum of serum iron level and UIBC ${ }^{14}$. Serum iron parameters evaluated with electrochemiluminescence method on the Roche Cobas 8000 modular analyzer.

\section{Statistical analyses}

Kolmogorov Smirnov test was used to check whether the continuous variables fit normal distribution. In the comparisons of more than two groups of normally distributed variables, ANOVA and LSD multiple comparison tests were conducted whereas Kruskal Wallis and Dunn multiple comparison test were used for the variables that did not fit normal distribution. Differences between repeated measurements were tested using paired samples $t$ test. Mean \pm std. deviation values were given as the de- scriptive statistics. SPSS for Windows version 22.0 package program was used for statistical analyses and $\mathrm{P}<0.05$ was accepted as the statistically significant value.

\section{Results}

Ferritin, serum iron level, UIBC and TIBC values of the rats in the Sp and S-by groups were compared to those of the rats in the Sh and C groups. It was observed that ferritin and serum iron levels did not change significantly in the S-by and Sp groups relative to the Sh and C groups ( $p>0.05)$. The statistically significant difference was seen UIBC values in the S-by and Sp groups than to the $C$ group $(\mathrm{p}<0.01)$. It was observed that the TIBC levels of $\mathrm{S}$-by and $\mathrm{Sp}$ groups decreased significantly in comparison to the $\mathrm{C}$ group $(\mathrm{p}<0.01)$ (Table 1$)$.

Table 1:

The comparision of ferritin, serum iron level, UIBC and TIBC values in the experiment groups.

\begin{tabular}{lcccc}
\hline Groups & $\begin{array}{c}\text { Ferritin } \\
(\mathbf{n g} / \mathbf{m L})\end{array}$ & $\begin{array}{c}\text { Serum iron level } \\
(\boldsymbol{\mu} \mathbf{g} / \mathbf{d L})\end{array}$ & $\begin{array}{c}\text { UIBC } \\
(\boldsymbol{\mu g} / \mathbf{d L})\end{array}$ & $\begin{array}{c}\text { TIBC } \\
(\boldsymbol{\mu g} / \mathbf{d L})\end{array}$ \\
\hline $\mathbf{C}(\mathrm{n}: 8)$ & $0.666 \pm 0.078$ & $223.3 \pm 46.4$ & $539.4 \pm 176.5$ & $762.6 \pm 202.4$ \\
Sh $(\mathrm{n}: 8)$ & $0.633 \pm 0.075$ & $219.1 \pm 51.1$ & $373.0 \pm 105.3^{*}$ & $592.1 \pm 124.4^{* *}$ \\
Sp $(\mathrm{n}: 8)$ & $0.589 \pm 0.072$ & $195.4 \pm 22.2$ & $350.0 \pm 36.0^{*}$ & $545.3 \pm 46.2^{*}$ \\
S-by $(\mathrm{n}: 8)$ & $0.610 \pm 0.030$ & $186.1 \pm 25.1$ & $361.5 \pm 66.5^{*}$ & $547.7 \pm 67.8^{*}$ \\
\hline C: Control group (n=8), Sh:Sham group (n=8), Sp: Speech group (n=8), S-by: Stand by group (n=8), UIBC: Unsaturated iron binding capacity, \\
TIBC: Total iron binding capacity, (Mean \pm SD) \\
" $p<0.01:$ Compared to C group, ${ }^{* *} p<0.05:$ Compared to C group.
\end{tabular}

\section{Discussion}

Deficiency or surplus of iron in the body leads to various diseases ${ }^{15}$. Iron deficiency negatively affects the immune system $^{16}$, thyroid metabolism ${ }^{17}$ and cognitive functions ${ }^{18}$. On the other hand, iron surplus is related to various pathological conditions such as cancer $^{19}$, neurodegenerative diseases ${ }^{20}$, and damage of $\operatorname{liver}^{21}$ and testicles ${ }^{22}$. Therefore, while keeping the serum iron levels and other iron parameters within a certain interval is important for body function, various complicated mechanisms take part in the establishment of this balance.

There are studies in the literature which show that EMF in different doses affect serum iron parameters. The implementation durations of EMF exposure and total experiment durations vary in these studies. Elferchichi et al. ${ }^{23}$ observed that a short 5-day exposure to a static magnetic field (SMF) of $128 \mathrm{mT}$ negatively affected serum iron levels. It was also seen that exposure to the SMF of the same dosage for three times longer (15 days) decreased the serum iron levels ${ }^{24}$. Fattahi et al. ${ }^{11}$, in their in vitro study, found that an exposure of 30 minutes to EMFs created by mobile phones decreased the levels of serum ferritin. They explained this decrease with the oxidative stress occurring because of EMF.

In addition to the studies where EMF is applied for short durations, there are also other studies where applications are made for longer durations. It was shown that serum ferritin and iron values were affected negatively by long exposure to $\mathrm{EMF}^{12,25}$. On the other hand, contrary to knowledge on the negative effects of EMF, there are also studies which showed that very low frequency EMF did not have an impact on serum iron levels ${ }^{4,26}$. In this study, serum iron and ferritin levels in experiment groups had a tendency to decrease, while there were not significant differences found. The insignificance of the difference between experiment groups and the control group may have been caused by the compensation of iron by iron storage areas in the body such as liver, spleen and bone marrow.

Transferrin is primarily formed by the bonding of apo-transferrin synthesized in the liver with $(\mathrm{Fe} 3+)^{27}$. UIBC represents the fraction of the transferrin protein that is not bonded with iron. There are few studies in the 
literature on the effects of EMF on transferrin. Djordjevich et al. ${ }^{28}$ found that the transferrin levels of rats exposed to SMF $(16 \mathrm{mT})$ for 28 days were increased. Likewise, there are studies which reported that short (5 to 15 days) exposure to $128 \mathrm{~m}$ T SMF increased the serum transferrin levels, while these studies found a negative relationship between transferrin and serum iron levels ${ }^{22,23}$. Contrary to previous results, in this study, it was observed that exposure to mobile phones working on EMF with $900 \mathrm{MHz}$ frequency, both in speech and stand by modes, significantly decreased UIBC which represents serum transferrin levels. It was seen that the difference between sham and control was also statistically significant. The limitations of the this study were that the parameters evaluated on the blood serum samples, the duration of experiment performed in a long period (10 week) and the occurring stress depending on the immobilization of rats for a long period. In connection to the significant decrease in UIBC, TIBC also decreased. The reason for the decrease in UIBC may be damage in the liver where transferrin is synthesized caused by the oxidative stress created by the EMF. Histopathologic investigation of the liver in further studies will be helpful in clearly revealing the activation mechanism between EMF and transferrin.

As a conclusion, mobile phone usage is becoming more and more widespread in the society. It is a necessity to investigate the potentially harmful effects of EMF created by mobile phones on different systems. In this study, it was determined that the EMF created by mobile phones on speech and standby did not affect serum iron and ferritin levels. On the other hand, it was observed that the EMF in both modes negatively affects UIBC. We suggest that these results will contribute to studies investigating the effects of EMF on hematologic parameters.

\section{Conflict of interest:}

The authors of this paper have no conflicts of interest, including specific financial interests, relationships, and/ or affiliations relevant to the subject matter or materials included.

\section{References}

1. Valberg PA, van Deventer TE, Repacholi MH. Workgroup report: Base stations and wireless networks-radiofrequency $(\mathrm{RF})$ exposures and health consequences. Environ Health Perspect 2007; 115: 416-424.

2. Mortavazi S, Habib A, Ganj-Karami A, Samimi-Doost R, Pour-Abedi A, Babaie A. Alterations in TSH and thy- roid hormones following mobile phone use. Oman Med J 2009; 24: 274-278.

3. Alghamdi MS, El-Ghazaly NA. Effects of exposure to electromagnetic field on some hematological parameters in mice. Open J Med Chem 2012; 2: 30-42.

4. Burchard JF, Nguyen DH, Block E. Macro- and trace element concentrations in blood plasma and cerebrospinal fluid of dairy cows exposed to electric and magnetic fields. Bioelectromagnetics 1999; 20: 358-364.

5. Richardson DR. Iron chelators as therapeutic agents for the treatment of cancer. Crit Rev Oncol Hematol 2002; 42; 267-281.

6. Fraga CG, Oteiza PI. Iron toxicity and antioxidant nutrients. Toxicology 2001; 180: 23-32.

7. Herbert V, Shaw S, Jayatilleke E. Vitamin C-driven free radical generation from iron. J Nutr 1996; 126: 1213-1220. 8. Murtagh LJ, Whiley M, Wilson S, Tran H, Bassett ML. Unsaturated iron binding capacity and transferrin saturation are equally reliable in detection of HFE hemochromatosis. Am J Gastroenterol 2001; 97(8): 2093-2099.

9. Podgórski T, Kryściak J, Konarski J, Domaszewska K, Durkalec-Michalski K, Strzelczyk R, Pawlak M. Iron metabolism in field hockey players during an annual training cycle. J Hum Kinet 2015; 47: 107-114.

10. AisenP. Studies on the binding of iron to transferrin and conalbumin. J Biol Chem 1966; 241: 1666-1671.

11. Fattahi-asl J, Baradaran-Ghahfarokhi M, Karbalae M, Baradaran-Ghahfarokhi M, Baradaran-Ghahfarokhi HR. Effects of radiofrequency radiation on human ferritin: An in vitro enzymun assay. J Med Signals Sens 2012; 2: 235240.

12. Hachulla E, Caulier-Leleu MT, Fontaine O, Mehianoui L, Pelerin P. Pseudo-iron deficiency in a French population living near high-voltage transmission lines: a dilemma for clinicians. Eur J Intern Med 2000; 11: 351-352. 13. Dasdag S, Ketani MA, Akdag Z, Ersay AR, Sari I, Demirtas ÖC, Celik MS. Whole-body microwave exposure emitted by cellular phones and testicular function of rats. Urol Res 1999; 27: 219-223.

14. Yamanishi H, Iyama S, Yamaguchi Y, Kanakura Y, Iwatani Y. Total iron-binding capacity calculated from serum transferrin concentration or serum iron concentration and unsaturated iron-binding capacity. Clin Chem 2003; 49: 175-178.

15. Nasri H, Nasri P, Baradaran-Ghahfarokhi M, Shahbazi-Gahrouei D, Fattahi-asl J. Mobile phone radiation and human serum components: A short literature review on recent findings. Life Sci J 2014; 11: 426-431. 
16. Ekiz C, Agaoglu L, Karakas Z, Gurel N, Yalcin I. The effect of iron deficiency anemia on the function of the immune system. Hematol J 2005; 5: 579-583.

17. Gökdeniz E, Demir C, Dilek İ. The effects of iron deficiency anemia on the thyroid functions. J Clin Exp Invest 2010; 1: 156-160.

18. Benton D. Micronutrient status, cognition and behavioral problems in childhood. Eur J Nutr 2008; 47: 38-50. 19. Beckman LE, Landeghem GFV, Sikström C, Vahlin A, Markevarn B, Hallmans G, Lenner P, Athlin L, Stenling R, Beckman L. Interaction between haemochromatosis and transferrin receptor genes in different neoplastic disorders. Carcinogenesis 1999; 20: 1231-1233.

20. Zatta P, Lucchini R, Rensburg SJV, Taylor A. The role of metals in neurodegenerative process: Aluminum, manganese and zinc. Brain Res Bull 2003; 62: 15-28.

21. Zhang Y, Huang Y, Deng X, Xu Y, Gao Z, Li H. Iron overload-induced rat liver injury: Involvement of protein tyrosine nitration and the effect of baicalin. Eur J Pharmacol 2012; 680: 95-101.

22. El-seweidy MM, Asker ME, Ali SI, Atteia HH. Effect of prolonged intake of iron enriched diet on testicular functions of experimental rats. Naturel Science 2010; 2: 551-556.
23. Elferchichi M, Abdelmelek H, Sakly M. Effects of sub-acute exposure to static magnetic field on iron status and hematopoiesis in rats. Turk J Hematol 2007; 24: 64-68. 24. Elferchichi M, Mercier J, Ammari M, Belguith H, Abdelmelek H, Sakly M, Lambert K. Subacute static magnetic field exposure in rat induces a pseudoanemia status withincrease in MCT4 and Glut4 proteinsin glycolytic muscle. Environ Sci Pollut Res 2016; 23: 1265-1273.

25. Nourmohammadi I, Ahmadvand H, Taghikhani M. Evaluation of levels of macro- and micro-nutrients in workers exposed to electromagnetic fields and comparison with levels of patients with leukemia. Iran Biomed J 2001; 5: 79-85.

26. Lotfi A, Shahryar HA, Narimani-Rad M. Effects of exposure to $50 \mathrm{~Hz}$ electromagnetic fields during incubation on some of serum biochemical measures in newly-hatched chicks. J Biol Environ Sci 2012; 6: 49-53.

27. Jandl JH. Blood: Pathophysiology, Boston, Blackwell Scientific Pub., 1991.

28. Djordjevich DM, De Luka SR, Milovanovich ID, Janković S, Stefanović S, Vesković-Moračanin S, Cirković S, Ilić AŽ, Ristić-Djurović JL, Trbovich AM. Hematological parameters' changes in mice subchronically exposed to static magnetic fields of different orientations. Ecotoxicol Environ Saf 2012; 81: 98-105. 